\title{
Modified Bell Tawse Technique for Correction of Pediatric Isolated Radial Head Dislocation without Ulnar Bowing
}

\section{Joana Monteiro Pereira*, Ana Esteves, Pedro Ribeiro, Francisco Bernardes, Tiago Costa and Pedro Barreira}

Centro Hospitalar Tâmega e Sousa, Penafiel, Portugal

*Corresponding Author: Joana Monteiro Pereira, Centro Hospitalar Tâmega e Sousa, Penafiel, Portugal.
Received: August 30, 2021

Published: September 18, 2021

(C) All rights are reserved by Joana Monteiro

Pereira., et al.

\section{Abstract}

Chronic radial head dislocation is a rare and potentially devastating injury in pediatric population, resulting in long term function and movement impairment. Early recognition and management are essential to restore elbow flexion and more important pronation and supination.

The treatment of chronic radial head dislocations in children can be challenging and currently there is no consensus on the optimal treatment.

The authors describe a case of a 6-year-old boy who suffered a fall onto his outstretched left arm, with 6 weeks evolution, and sustained a left radial head fracture with anterior dislocation, without ulnar deformity. A Boyd and Speed approach was made, and a modified Bell Tawse procedure was performed using a lateral slip of the triceps tendon, passed around the radial neck and fixed with a suture anchor into ulna.

This versatile approach allowed access to all the structures, minimizing the risk of surgical scarring, with excellent functional and imagiologic results, without long-term major complications.

To our knowledge, there are no reported cases regarding an annular ligament reconstruction using a modified Bell Tawse procedure with suture anchor.

Level of evidence IV.

Keywords: Bell Tawse Technique; Radial Head Dislocation; Ulnar Bowing

\section{Introduction}

Isolated traumatic radial head dislocations are rare in children [1] and oftenoccur inpatients with a history of Monteggia fractures [2]. Although most children are at first asymptomatic, complaints may arise over the course of years. Restricted motion, cubitus deformity, pain, degenerative arthritis, and palsy can occur in this lesion [3].
Chronic post-traumatic radial head dislocation is defined as traumatic dislocation of the radial head over 4 weeks, caused generally by missed diagnosis or ineffective treatment of acute phase $[4,5]$.

The annular ligament plays a fundamental role in maintaining the position of the radial head and the stability of the elbow joint 
[2] and several treatment strategies have been proposed, including a conservative approach [6] and open reduction of the radial head along with reconstruction of the annular ligament and/or ulnar osteotomy to maintainthe forearm rotational stability [2].

Numerous complications of such procedures have been reported which include subluxation and redislocation, stiffness, elbow instability, osteotomies nonunion, avascular necrosis (AVN) of the radial head, nerve injury, degenerative arthritis, and infection $[7,8]$.

We present a case of a missed radial head dislocation and its subsequent management using a Modified Bell Tawse procedure with excellent results and no long- term complications.

\section{Case Report}

A 6-year-old boy presented to our emergency department following a fall onto his outstretched left arm, with 6 weeks of evolution.

He sustained a left radial head fracture with anterior dislocation, without ulnar deformity (Figure 1 and 2). He had pain complaints at the elbow with deficit of $20^{\circ}$ flexion, $20^{\circ}$ extension and $20^{\circ}$ supination. On physical examination, he also presented $15^{\circ} \mathrm{cu}-$ bitus valgus (Figure 3).

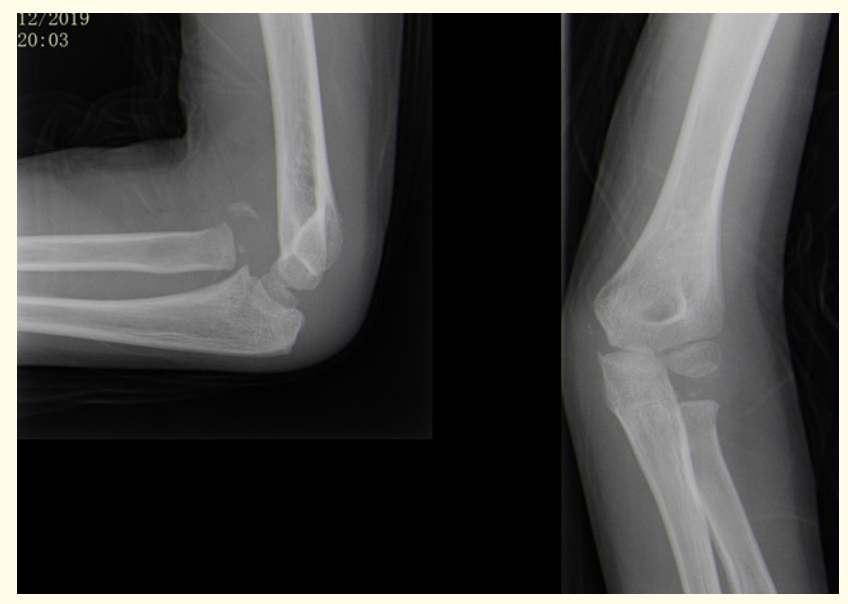

Figure 1: X-ray (AP and Lateral view) showing anterior radial head dislocation with radiocapitellar line disruption and anterior fragment resulting from radial head fracture.

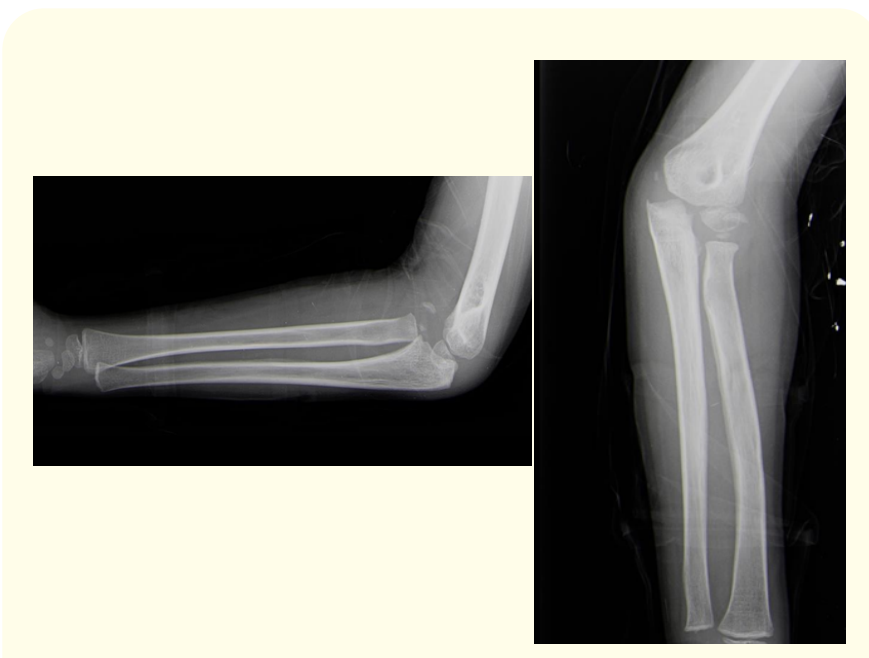

Figure 2: X-ray (AP and Lateral view) showing no ulnar deformity (bowing or fracture).

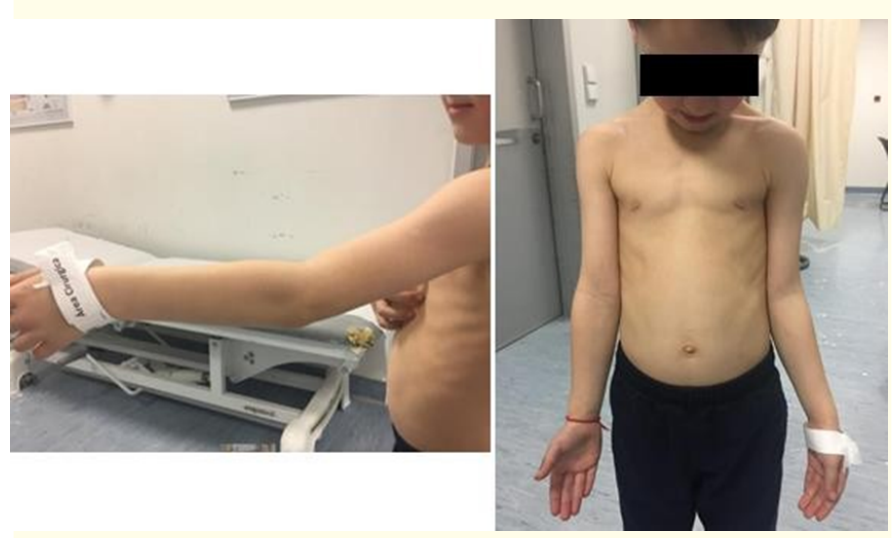

Figure 3: 6-year-old boy with $20^{\circ}$ extension deficit and $15^{\circ}$ valgus deformity.

CT showed anterior dislocation of the proximal end of the radius, with radial head fracture (Figure 4).

The MRI demonstrated anterior dislocation of the radius with annular ligament rupture (Figure 5). In intra-articular topography, an osseocartilaginous fragment is presented slightly cranial and anterior to the proximal end of the radius is observed, with approx- 


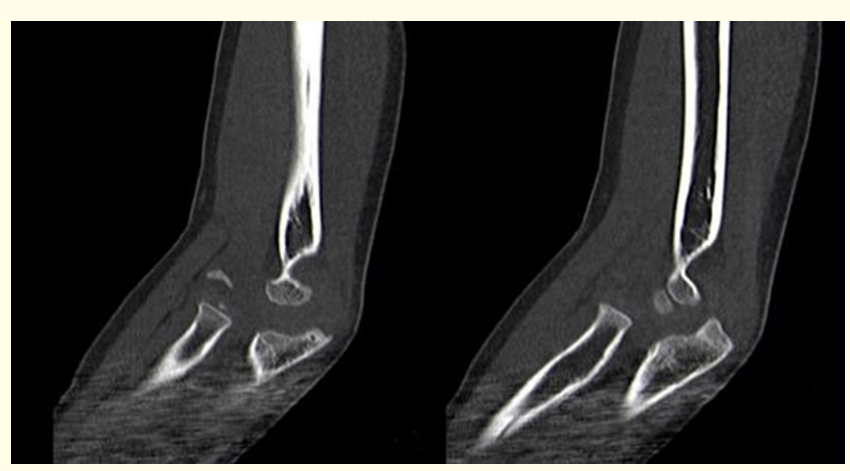

Figure 4: CT (Sagittal cut) showing fragment resulting from radial head fracture and anterior radial head dislocation.

imately $9 \times 8 \mathrm{~mm}$. Integrity of the internal and external collateral ligaments of the elbow.

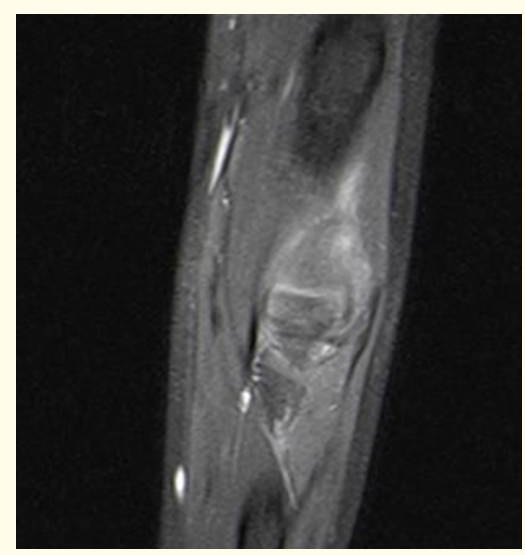

Figure 5: MRI (Coronal cut) showing annular ligament disrupted.

We performed an open reduction through a Boyd and Speed approach. This approach has the advantage of harvesting the triceps tendon and reduction of the radial head using a single incision.

After joint capsule identification, the annular ligament, which was ruptured and obstructing the radial head reduction, was located. Then, the intra-articular space was cleared of debris by removing any fragments of interposed capsule. Reduction of the ra- dial head was verified (Figure 6) and ligament reconstruction was undertaken.

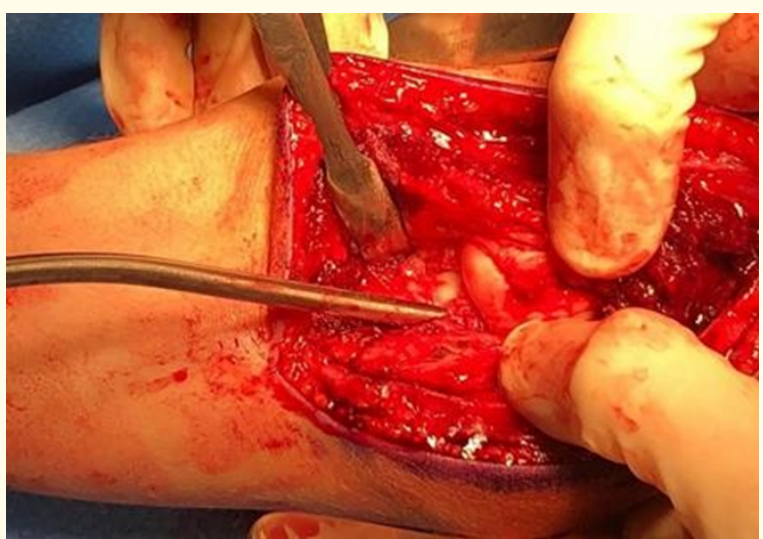

Figure 6: Intra operative images showing radial head reduction.

For annular ligament reconstruction, a 10-cm lateral long slip of triceps tendon was fashioned (modified Bell Tawse procedure) of $0.5 \mathrm{~cm}$ width from proximal to distal. Care is taken to not detach its insertion at the ulna. The re-insertion of triceps tendon was made with a suture anchor (Micro Corkscrew 2.2 Arthrex) after passing it round radius neck, from posterior to anterior (Figure 7). The radial head reduction was confirmed through intraoperative fluoroscopy.

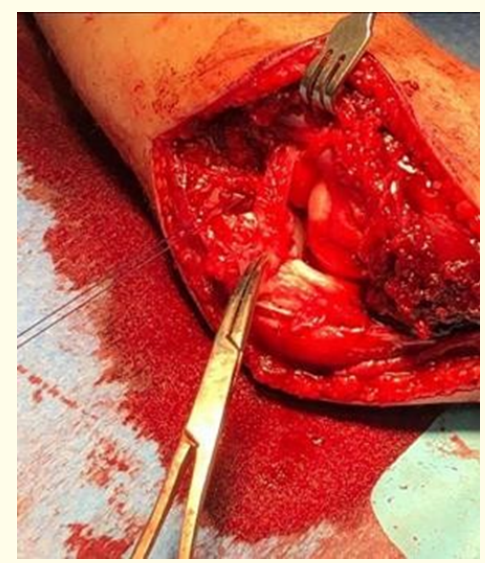

Figure 7: Intra operative images showing lateral triceps slip around radial neck and reduction of radial head. 
A long-arm cast was applied for three weeks, with the forearm positioned at $90^{\circ}$ of flexion and supination. After the removal of the cast, self-managed forearm pronation and supination was recommended for two weeks, followed by rehabilitation exercises for elbow flexion and extension two weeks later.

The postoperative radiographs showed good reduction of the dislocation of the radial head (Figure 8).

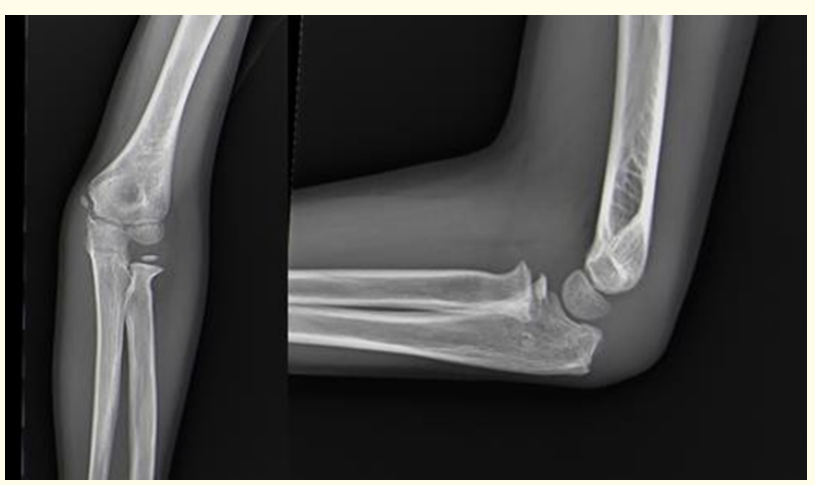

Figure 8: Follow-up X-ray (AP and Lateral view) showing radial head reduction, without imagiologic complications.

At 1-year of follow-up the patient remains symptoms-free with a well-healed scar. On examination we presented with complete mobility, without extension/flexion and pronation/supination deficits (Figure 9). Clinically with valgus correction (Figure 10).

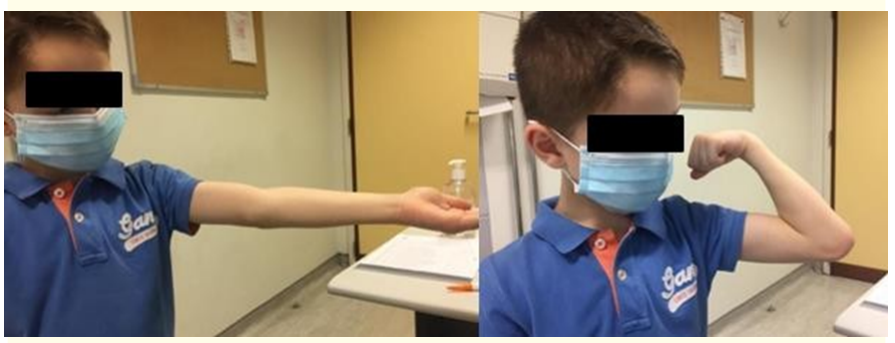

Figure 9: At 1-year follow-up: Elbow without flexion or extension deficit.

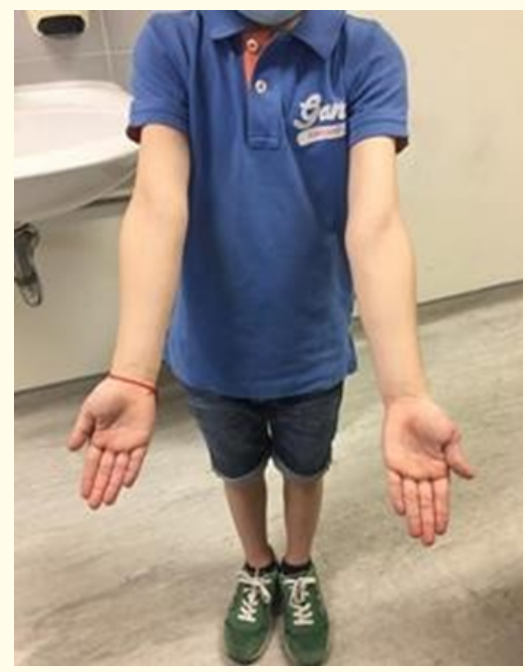

Figure 10: At 1 year-follow-up: Elbow with valgus deformity correction.

MRI showed integrity of the annular neo-ligament.

\section{Discussion}

Post-traumatic dislocation of the radial head without an associated fracture of the ulna is uncommon and easily overlooked. Most of the radial dislocation was overlooked on the original radiographs due to the subtle radiologic signs. A careful evaluation of the radiographs is important to a correct diagnosis [9].

A missed diagnosis of acute dislocation results in chronic dislocation [3]. In this case, the patient presented to our department with a chronic dislocation of the radial head with an initial trauma six weeks ago.

According to Lincoln and Mubarak, the term isolated traumatic dislocation of the radial head may be a misnomer and suggested that the injury was more accurately described as a minimal Monteggia fracture-dislocation (often associated with plastic bowing of the ulna) [10]. They recommended that the maximum ulnar bow should be measured to detect ulnar bowing. In cases of apparent bowing of the ulna, osteotomy may be necessary and ligament reconstruction alone would be insufficient to stabilize the radial 
head [10]. In our case, the ulnar bowing is not presented, and ulnar osteotomy was not required.

Several studies have shown that the annular ligament plays an important role in maintaining the stability of the proximal radioulnar and radiocapitellar joints of the elbow [11,12]. Cohen., et al. shown that lateral elbow stability was provided by the annular ligament [11]. These evidences highlight the importance of the annular ligament in maintaining normal elbow kinematics by functioning as a constraint of the radial head [2].

The annular ligament could be reconstructed by using triceps tendon, triceps fascia, antebrachial fascia, palmaris longus tendon, or surgical thread. However, each of these tissues has drawbacks. The forearm fascia and tendon of the palmaris longus are too weak to restrict the radius, and an additional incision is required for harvesting the fascia lata. Non-absorbable artificial suture materials, such as nylon, are unyielding and cannot accommodate the continued radial growth, thereby possibly leading to the narrowing of the radial neck [14-16].

Bell Tawse described the reconstruction of the annular ligament by turning down a slip of the triceps tendon, leaving it attached to the ulna, and passing it around the neck of the radius from behind forward and securing it through a drill hole in the ulna [2]. LloydRoberts and Bucknill introduced a modification to the Bell Tawse technique: they used a lateral triceps tendon bundle that they lowered to the level of the radial neck to reconstruct the annular ligament [17].

Optimal fixation devices should confer immediate stability, resist gap formation, promote biologic healing, and restore the anatomic footprint of the native ligament [18]. Suture anchor designs allow direct implant insertion (without the need for punching, tapping or predrilling), produce tension throughout the tendon-bone interface (biomechanically superior construct) [19], less surgical dissection and decrease operative time compared with bone tunnel. The use of suture anchor in this case support use of an anchor technique as a viable alternative to transosseous repair of the annular ligament.

Complications are common after surgical treatment and include subluxation, redislocation, stiffness, elbow instability, osteotomies nonunion, avascular necrosis of the radial head, nerve injury, degenerative arthritis, and infection $[7,8]$.
Complications like osteolytic changes, narrowing or growth disturbance of the radial neck, may arise due to excessive pressure by the newly reconstructed ligament on the radial neck $[20,21]$. An important consideration when reconstructing the annular ligamentis to avoid excessive tension as this may restrict rotation of the radial neck.

In this case, we performed a modified Bell Tawse procedure and used a lateral slip of the triceps tendon, passed around the radial neck and fixed with a suture anchor in the ulna, with excellent functional and imagiologic results, without complications.

\section{Conclusion}

In this clinical case, it is important to highlight the rarity of the lesion in pediatric population.

Pediatric cases of isolated radial head dislocation can be successfully managed with minimal complications by using Bell Tawse modified technique for the reconstruction of the annular ligament.

There are several techniques which can be used alone or in combination to achieve relocation of the chronically dislocated radial head depending on the intraoperative findings and stability.

This procedure has several advantageous because of the use of a sturdy, well vascularized tendon, which allows the complete reconstruction of annular ligament, to stabilize the head of the radius within the elbow joint.

To our knowledge, there are no reported cases regarding a modified Bell Tawse procedure using suture anchor to fix the ligament at ulna.

\section{Statement of Ethics}

The paper is exempt from ethical committee approval. Written informed consent was obtained from the parents of the patient for publication of this case report and any accompanying images and validated at the hospital.

\section{Conflict of Interest Statement}

The authors have no conflicts of interest to declare.

\section{Funding Sources}

The authors have no funding sources to declare. 


\section{Bibliography}

1. Kim HT., et al. "Chronic radial head dislocation in children, Part 1: pathologic changes preventing stable reduction and surgical correction". Journal of Pediatric Orthopaedics 22 (2002): 583.

2. Lei Tan., et al. "Modified technique for correction of isolated radial head dislocation without apparent ulnar bowing: a retrospective case study". International Journal of Clinical and Experimental Medicine 8.10 (2015): 18197-11820.

3. Ruchelsman DE., et al. "Persistent posterior interosseous nerve palsy associated with a chronic type I Monteggia fracture-dislocation in a child: a case report and review of the literature". Hand 4 (2009): 167-172.

4. Horii E., et al. "Surgical treatment for chronic radial head dislocation". Journal of Bone and Joint Surgery American 84-A (2002): 1183-1188.

5. Bhaskar A. "Missed Monteggia fracture in children: Is annular ligament reconstruction always required?" The Indian Journal of Orthopaedics 43 (2009): 389-395.

6. Thompson JD and Lipscomb AB. "Recurrent radial head subluxation treated with annular ligament reconstruction: A case report and follow-up study". Clinical Orthopaedics and Related Research 246 (1989): 131.

7. Futami T., et al. "Rotation osteotomy for dislocation of the radial head 6 cases followed for 7 (3-10) years". Acta Orthopaedica Scandinavica 63 (1992): 455-456.

8. Mehta SD. "Flexion osteotomy of ulna for untreated". Strategies in Trauma and Limb Reconstruction 2.1 (2007): 31-34.

9. Wang MN and Chang WN. "Chronic posttraumatic anterior dislocation of the radialhead in children: thirteen cases treated by open reduction, ulnar osteotomy, and annular ligament reconstruction through a Boyd incision". Journal of Orthopaedic Trauma 20 (2006): 1-5.

10. Inoue G and Shionoya K. "Corrective ulnar osteotomy for malunited anterior Monteggia lesions in children: 12 patients followed for 1-12 years". Acta Orthopaedica Scandinavica 69 (1998): 73-76.

11. Cohen MS and Hastings H. "Rotatory Instability of the Elbow. The Anatomy and Role of the Lateral Stabilizers". Journal of Bone and Joint Surgery American 79 (1997): 225-233.
12. Søjbjerg J., et al. "The stability of the elbow following excision of the radial head and transection of the annular ligament". Archives of Orthopaedic and Trauma Surgery 106 (1987): 248250.

13. Dunning CE., et al. "Ligamentous stabilizers against posterolateral rotatory instability of the elbow". Journal of Bone and Joint Surgery American 83 (2001): 1823-1828.

14. Ghinea CA., et al. "Burnei's technique in the treatment of radial head displacement; innovative surgery: Study on two cases". The Journal of Medicine and Life 6 (2013): 26-33.

15. Rodgers WB., et al. "Chronic Monteggia lesions in children: Complications and results of reconstruction". Journal of Bone and Joint Surgery American 78 (1996): 1322-1329.

16. Fowles JV., et al. "The Monteggia lesion in children: Fracture of the ulna and dislocation of the radial head". Journal of Bone and Joint Surgery American 65 (1983): 1276.

17. Lloyd-Roberts $\mathrm{G}$ and Bucknill T. "Anterior dislocation of the radial head in children: aetiology, natural history and management". The Journal of Bone and Joint Surgery. British 59 (1977): 402.

18. Brian J Cole., et al. "Fixation of Soft Tissue to Bone: Techniques and Fundamentals". The Journal of the American Academy of Orthopaedic Surgeons 24.2 (2016): 83-95.

19. Park MC., et al. "Tendon-to-bone pressure distributions at a repaired rotator cuff footprint using transosseous suture and suture anchor fixation techniques". American Journal of Sports Medicine 33.8 (2005): 1154-1159.

20. Thompson JD and Lipscomb AB. "Recurrent radial head subluxation treated with annular ligament reconstruction: A case report and follow-up study". Clinical Orthopaedics and Related Research 246 (1989): 131.

21. Galik K., et al. "The effect of the annular ligament on kinematics of the radial head". Journal of Hand Surgery 32 (2007): 1218-1224.

\section{Volume 4 Issue 10 October 2021 (C) All rights are reserved by Joana Monteiro Pereira., et al.}

